\title{
SYSTEMS OF $n$ PARTIAL DIFFERENTIAL EQUATIONS IN $n$ UNKNOWN FUNCTIONS: THE CONJECTURE OF M. JANET
}

BY

\author{
JOSEPH JOHNSON
}

\begin{abstract}
It was conjectured by Janet that an analytic solution to a system of $n$ "independent" analytic differential equations in $n$ unknown functions if not isolated must depend on at least one unknown function of $m-1$ variables plus possibly other functions of fewer than $m$ variables. Here $m$ is the dimension of the complex domain on which the equations and the solution are given. An algebraic generalization of the linear form of the conjecture is proven. Also the result is extended to give a nonlinear version.
\end{abstract}

The purpose of this note is to show that the conjecture of Maurice Janet stated in [1] is a corollary of a result by Goodearl (Theorem 7 of [2]). That is done in Theorem 1. In Theorem 2, a nonlinear generalization of Janet's conjecture is proved. The reader who would like to know in advance the original form of Janet's conjecture should read the corollary to Theorem 2 and the discussion that follows the corollary.

1. Goodearl's result. If $\Delta$ is a finite set and $R$ a differential ring (possibly noncommutative) with $\Delta$ as its fundamental set of (commuting) derivation operators we will call $R$ a $\Delta$-ring. In fact generally we will write " $\Delta$ " where formerly we would have written "differential." Let us write $\mathscr{D}_{R}$ for the ring of linear $\Delta$-operators with coefficients in $R$. We will think of the elements of $\mathscr{D}_{R}$ as polynomials in $\Delta$ with coefficients in $R$ written on the left. Naturally in $\mathscr{D}_{R}$, if $1 \leqslant i, j \leqslant m$ and $a \in R, \delta_{i} \delta_{j}=\delta_{j} \delta_{i}$ and $\delta_{i} a=\delta_{i}(a)+a \delta_{i}$.

For the reader's convenience, the following paraphrase of Goodearl's result is given here. In its statement pd stands for "projective dimension."

THEOREM (GOODEARL). Let the $\Delta$-ring $R$ be semiprime and right and left noetherian. If $M$ is a nonzero right $\mathscr{D}_{R}$-module and finitely generated as a right $R$-module then $\operatorname{pd}_{\oplus_{R}} M=$ card $\Delta+\operatorname{pd}_{R} M$.

Let $m \in \mathbf{N}$ and fix a set $\Delta$ with $m$ elements $\delta_{1}, \ldots, \delta_{m}$. Let $k$ be a

Received by the editors April 20, 1977.

AMS (MOS) subject classifications (1970). Primary 12H05.

Key words and phrases. Differential algebra, differential ring, differential module, Kähler differentials. 
commutative $\Delta$-field and $M$ a finitely generated $\Delta$-vector space over $k$ (= left $\mathscr{D}_{k}$-module). For $r$ in $\mathbf{N}$ let $\mathscr{D}_{r}$ be the elements of $\mathscr{D}_{k}$ of order $\leqslant r$. Let $x_{1}, \ldots, x_{n}$ be a set of generators of $M$ and $\mathbf{r}$ an indeterminate. There is a polynomial $\chi(\mathbf{r}) \in \mathbf{Q}[\mathbf{r}]$ (cf. [3, Theorem on p. 241]) such that $\chi(r)=$ $\operatorname{dim}_{k}\left(\mathscr{D}_{r} x_{1}+\cdots+\mathscr{D}_{r} x_{n}\right)$ for all sufficiently large $r$ in $\mathbf{N}$. The degree $\tau(M)$ of $\chi(\mathbf{r})$ does not depend on the choice of $x_{1}, \ldots, x_{n}$. We call $\tau(M)$ the type of $M$. Always $\tau(M) \leqslant m$. If $M=0$, define $\tau(M)=-1$.

THEOREM 1. Let $k$ be an infinite $\Delta$-field and $n \in \mathbf{N}$. Consider any exact sequence $0 \rightarrow \mathscr{Q}_{k}^{n} \rightarrow \mathscr{Q}_{k}^{n} \rightarrow M \rightarrow 0$ of left $\mathscr{D}_{k}$-modules. If $\tau(M)<m-1$, then $M=0$.

This form of Janet's conjecture is the one that follows most quickly from [2]. As it does not appear in the literature, the original form of the conjecture will be derived from it at the end of this note (see Corollary to Theorem 2).

Let $C$ be the field of constants of $k$ and $c \in \mathrm{GL}(m, C)$. Let $\varepsilon_{i}=\sum_{j=1}^{m} c_{i j} \delta_{j}$ and consider $\varepsilon_{1}, \ldots, \varepsilon_{m}$ in the obvious way as derivation operators of $k$. Then $k$ is an $\left\{\varepsilon_{1}, \ldots, \varepsilon_{m}\right\}$-field and $M$ is an $\left\{\varepsilon_{1}, \ldots, \varepsilon_{m}\right\}$-vector space over $k$. It is clear that replacing $\delta_{1}, \ldots, \delta_{m}$ by $\varepsilon_{1}, \ldots, \varepsilon_{m}$ does not change the polynomial $\chi(r)$. The theorem on p. 212 of [4] implies the following.

LEMMA 1. Let $k$ be an infinite $\Delta$-field and $M$ a finitely generated $\Delta$-vector space over $k$. If $0 \leqslant h \leqslant m$ and $\tau(M) \leqslant h, c$ can be chosen so that $M$ is finitely generated as an $\left\{\varepsilon_{1}, \ldots, \varepsilon_{h}\right\}$-vector space over $k$.

To prove the theorem let us assume $\tau(M)<m-1$ but $M \neq 0$ and show a contradiction result. By Lemma 1 we can assume $M$ is finitely generated as a $\left\{\delta_{1}, \ldots, \delta_{m-2}\right\}$-vector space over $k$. Let $R$ be the subring of $\mathscr{D}_{k}$ generated by $k$ and $\delta_{1}, \ldots, \delta_{m-2}$. Then $M$ is finitely generated as a left $R$-module since $R$ is the ring of linear $\left\{\delta_{1}, \ldots, \delta_{m-2}\right\}$-operators over $k$. Also $R$ is a $\left\{\delta_{m-1}, \delta_{m}\right\}$-ring with

$$
\delta_{i}\left(a \delta_{1}^{r_{1}} \cdots \delta_{m-2}^{r_{m-3}}\right)=\delta_{i}(a) \delta_{1}^{r_{1}} \cdots \delta_{m-2}^{r_{m-3}}
$$

for $i=m-1, m, a \in k$ and $r_{1}, \ldots, r_{m-2} \in \mathbf{N}$. We have $\mathscr{D}_{k}=\mathscr{D}_{R}$. Lemma 2 below will show that Theorem 7 of [2], which is stated for right modules, is valid for left modules also. When it is applied to our situation it says $\operatorname{pd}_{\oplus_{k}} M=2+\operatorname{pd}_{R} M$. Clearly $\operatorname{pd}_{\Phi_{k}} M<1$ so that is a contradiction.

If $R$ is any ring, $R^{0}$ will represent the opposite ring of $R$. Also if $a \in R$, we will write $a^{0}$ whenever we want to consider $a$ as an element of $R^{0}$.

LeMma 2. Let $R$ be $a \Delta$-ring and consider $R^{0}$ as a $\Delta$-ring by $\delta\left(a^{9}\right)=$ $-(\delta(a))^{0}$ whenever $\delta \in \Delta$ and $a \in R$. Then we have a canonical isomorphism $\left(\mathscr{D}_{R}\right)^{0} \approx \mathscr{D}_{R^{0}}$

Let $\Theta$ denote the set of all monomials $\theta=\delta_{1}^{r_{1}} \cdots \delta_{m}^{r_{m}}$ with $r_{1}, \ldots, r_{m} \in$ 
$\mathbf{N}$ and define ord $\theta=r_{1}+\cdots+r_{m}$. Let $\alpha_{R}: \mathscr{D}_{R} \rightarrow \mathscr{D}_{R^{0}}$ be the unique additive map with $\alpha_{R}(a \theta)=\theta a^{0}$ whenever $\alpha \in R$ and $\theta \in \Theta$. It will suffice to show that if $D, E \in \mathscr{D}_{R}$, then $\alpha_{R}(D E)=\alpha_{R}(E) \alpha_{R}(D)$, since then it is clear that $\alpha_{R^{0}}:\left(\mathscr{D}_{R^{0}}\right)^{0} \rightarrow \mathscr{D}_{R}$ is inverse to $\alpha_{R}$.

For simplicity let us write $\alpha$ for $\alpha_{R}$. It is enough to show that if $a, b \in R$, $\theta, \phi \in \Theta$, then $\alpha(a \theta b \phi)=\alpha(b \phi) \alpha(a \theta)$. First note $\alpha(a b \phi)=\phi(a b)^{0}=\left(\phi b^{0}\right) a^{0}$ $=\alpha(b \phi) \alpha(a)$, so this holds when ord $\theta=0$. Let ord $\theta>0$, and write $\theta=\theta^{\prime} \delta$ with $\delta$ in $\Delta$. By using induction on ord $\theta$ we see that

$$
\begin{aligned}
\alpha(a \theta b \phi) & =\alpha\left(a \theta^{\prime}(\delta(b)+b \delta) \phi\right)=\alpha(\delta(b) \phi) \alpha\left(a \theta^{\prime}\right)+\alpha(b \delta \phi) \alpha\left(a \theta^{\prime}\right) \\
& =\phi\left[(\delta(b))^{0}+\delta b^{0}\right] \theta^{\prime} a^{0}=\phi\left[(\delta(b))^{0}+\delta\left(b^{0}\right)+b^{0} \delta\right] \theta^{\prime} a^{0} \\
& =\phi b^{0} \delta \theta^{\prime} a^{0}=\alpha(b \phi) \alpha(a \theta)
\end{aligned}
$$

so Lemma 2 is proved.

For later use, for each $r$ in $\mathbf{N}$ let us define $\Theta_{r} \subset \Theta$ to be all elements $\theta$ of $\Theta$ with ord $\theta<r$.

2. Generalization to nonlinear systems. Let $A$ be any commutative ring and $M$ an $A$-module. Let $\mathcal{H}=\left(H_{i}\right)_{i \in I}$ be a family of elements of $M$. If $\mathfrak{p} \in$ $\operatorname{Spec} A$ call $\mathcal{H}$ independent at $\mathfrak{p}$ if the family $\left(H_{i}+\mathfrak{p} M\right)$ of $M / \mathfrak{p} M$ is linearly independent over $A / \mathfrak{p}$.

LEMMA 3. Let $M$ be a free A-module, $\mathcal{H}$ a family of elements of $M$. Let $\mathfrak{p}, \mathfrak{q} \in \operatorname{Spec} A$ with $\mathfrak{p} \supset \mathfrak{q}$. If $\mathcal{H C}$ is independent at $\mathfrak{p}$, it is independent at $\mathfrak{q}$ also.

We can easily reduce to the case of a finite family $H=\left\{H_{1}, \ldots, H_{p}\right\}$ in a module $M$ which is free on a finite set of generators $e_{1}, \ldots, e_{Q}$. Write $H_{i}=\sum_{j=1}^{Q} h_{i j} e_{j}$ for $i=1, \ldots, P$ where $h_{i j} \in A$ for $j=1, \ldots, Q$. Independence of $\mathcal{H}$ at $\mathfrak{p}$ means a $P \times P$-submatrix of $\left(h_{i j}\right)_{1<i<P, 1 \leqslant j \leqslant Q}$ has its determinant $D$ not in $\mathfrak{p}$. Then $D \notin q$ either so $H$ is independent at $q$.

Let $k$ be a $\Delta$-field, $y_{1}, \ldots, y_{n} \Delta$-indeterminates and let $A=k\left\{y_{1}, \ldots, y_{n}\right\}$. Then $\Omega_{A / k}$ (the module of Kähler 1-forms of $A$ over $k$ ) is a free $A$-module on $d_{A / k}\left(\Theta y_{1} \cup \cdots \cup \Theta y_{n}\right)$. Also $\Omega_{A / k}$ is a $\mathscr{D}_{A}$-module in such a way that $d_{A / k}(\delta P)=\delta\left(d_{A / k} P\right)$ for every $P$ in $A$ and every $\delta$ in $\Delta$. Let $F_{1}, \ldots, F_{h} \in A$ and $\mathfrak{p} \in \operatorname{Spec} A$. Call $F_{1}, \ldots, F_{h}$ independent at $\mathfrak{p}$ if the family $\Theta d_{A / k} F_{1}$ $\cup \cdots \cup \Theta d_{A / k} F_{h}$ is. Then necessarily $h \leqslant n$. Also if $\mathfrak{p} \supset \mathfrak{q}$ are prime ideals of $A$ and $F_{1}, \ldots, F_{h}$ is independent at $\mathfrak{p}$, then by Lemma $3 F_{1}, \ldots, F_{h}$ is independent at $q$ also.

If $q$ is a prime $\Delta$-ideal of $A$ define $\tau(q)=\tau\left(\Omega_{K / k}\right)$ where $K$ is the quotient field of $A / \mathrm{q}$. Call q a separable prime $\Delta$-ideal of $A$ (or just separable) if $K$ is a separable extension of $k$.

THEOREM 2. Let $k$ be an infinite $\Delta$-field, $y_{1}, \ldots, y_{n} \Delta$-indeterminates over $k$, 
$A=k\left\{y_{1}, \ldots, y_{n}\right\}$. Let $F_{1}, \ldots, F_{n} \in A, \mathfrak{a}=\left[F_{1}, \ldots, F_{n}\right]$ and let $\mathfrak{p}$ be $a$ prime ideal of $A$ with $\mathfrak{a} \subset \mathfrak{p}$. Let $\mathrm{q}=\{a \in A: a(A \backslash \mathfrak{p}) \cap \mathfrak{a} \neq \varnothing\}$. Assume $F_{1}, \ldots, F_{n}$ are independent at $\mathfrak{p}$. Then:

(1) $\mathfrak{q}$ is the unique minimal prime component of $\mathfrak{a}$ contained in $\mathfrak{p}$ and is a separable ideal of $A$;

(2) Either $\tau(q)=m-1$ or $\tau(q)=-1$;

(3) If $\tau(q)=-1, q$ is a maximal ideal of $A$ (so $q=\mathfrak{p}$ and $\mathfrak{p}$ is then a $\Delta$-ideal) and $A / \mathfrak{q}$ is a finitely generated separably algebraic extension of $k$.

Let $\subseteq=A_{\mathfrak{p}}, \mathfrak{m}=\mathfrak{p} \subseteq$. For any $r$ in $\mathbf{N}$ let $\varrho_{r}$ be the local $k$-subalgebra of $\mathcal{D}$ generated by $B_{r}=\Theta_{r} y_{1} \cup \cdots \cup \Theta_{r} y_{n}$ and $\mathfrak{m}_{r}$ its maximal ideal $\varrho_{r} \cap \mathfrak{m}$. Now to establish (1) it is enough to show that $\mathcal{O a}_{\text {a }}$ a prime ideal of $\mathscr{D}$ and is separable. This will follow once we show that whenever $G_{1}, \ldots, G_{N}$ is a finite subset of $\Theta F_{1} \cup \cdots \cup \Theta F_{n}$ and $r$ is chosen so that $G_{1}, \ldots, G_{N} \in \searrow_{r}$, then

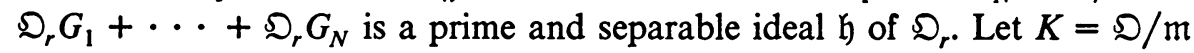
and $K_{r}=\varrho_{r} / \mathfrak{m}_{r}$. We have a $K_{r}$-linear map

$$
i: \mathfrak{m}_{r} / \mathfrak{m}_{r}^{2} \rightarrow K \otimes_{A} \Omega_{A / k}
$$

defined by $i\left(a+\mathfrak{m}_{r}^{2}\right)=1 \otimes d_{A / k}(a)$ whenever $a \in \mathfrak{m}_{r}$. Our hypothesis that $F_{1}, \ldots, F_{n}$ are independent at $\mathfrak{p}$ implies that $1 \otimes d_{A / k} G_{1}, \ldots, 1 \otimes d_{A / k} G_{N}$ (in $K \otimes_{A} \Omega_{A / k}$ ) are linearly independent over $K$. It follows that in $\mathfrak{m}_{r} / \mathfrak{m}_{r}^{2}$, $G_{1}+\mathfrak{m}_{r}^{2}, \ldots, G_{N}+\mathfrak{m}_{r}^{2}$ are linearly independent over $K_{r}$. By [5, Proposition 22, p. IV-40] $G_{1}, \ldots, G_{N}$ generate a prime ideal of $\mathcal{D}_{r}$.

To prove $\mathfrak{h}$ is separable let $L$ be the quotient field of $\mathfrak{D}_{r} / \mathfrak{h}$ and let $H=\operatorname{card} B_{r}$. If $u \in \mathcal{D}_{r}$, let $\bar{u}$ be its canonical image in $L$. Now $\sum_{v \in B_{r}}\left(\partial G_{i} / \partial v\right)-d_{L / k} \bar{v}=0$ for $i=1, \ldots, N$ is a set of $N$ linearly independent relations (over $L$ ) on $d_{L / k}\left(B_{r}\right)$ and also $d_{L / k}\left(B_{r}\right)$ itself generates $\Omega_{L / k}$ as a vector space over $L$. Therefore $\operatorname{tr} \operatorname{deg}_{k} L \geqslant N-H \geqslant \operatorname{dim}_{L} \Omega_{L / k}$. By Lemma 5 of $\S 1$ of [6], $L$ is a separable extension of $k$.

To prove (2), define $\subseteq=A_{q}, \mathfrak{m}=q \mathfrak{Q}$ and $K=\subseteq / \mathfrak{m}$. Now $F_{1}, \ldots, F_{n}$ are independent at q. Therefore if $\omega_{j}=1 \otimes d_{A / k} F_{j} \in K \otimes_{A} \Omega_{A / k}$, then $\omega_{1}, \ldots, \omega_{n}$ are linearly independent over $\mathscr{D}_{K}$. We have a well-known exact sequence of $\Delta$-vector spaces

$$
\mathrm{m} / \mathrm{m}^{2} \stackrel{i}{\rightarrow} K \otimes_{A} \Omega_{A / k} \rightarrow \Omega_{K / k} \rightarrow 0 .
$$

In this instance $i$ must be injective because $\mathrm{m} / \mathrm{m}^{2}$ is generated as a left $\mathcal{D}_{K}$-module by $F_{1}+\mathrm{m}^{2}, \ldots, F_{n}+\mathfrak{m}^{2}$. Hence we have an exact sequence of the form

$$
0 \rightarrow \mathscr{D}_{K}^{n} \rightarrow \mathscr{D}_{K}^{n} \rightarrow \Omega_{K / k} \rightarrow 0 .
$$

Therefore by Theorem 1 (with $K$ in place of $k$ ), $\tau\left(\Omega_{K / k}\right)=m-1$ or $\tau\left(\Omega_{K / k}\right)$ $=-1$. Since $\tau(\mathrm{q})=\tau\left(\Omega_{K / k}\right)$ this proves (2).

Let $K$ be the quotient field of $A / q$ and for all $r$ in $\mathbf{N}$ let $K_{r}$ be the field 
extension of $k$ which is generated by the image in $K$ of $\Theta_{r} y_{1} \cup \cdots \cup \Theta_{r} y_{n}$. Now if $\tau(q)=-1$ then $\Omega_{K / k}=0$. Hence by Theorem 5 in $\S 6$ of [6], $\Omega_{K_{r} / k}=0$ for all sufficiently large $r$ in N. Since $K_{r}$ is a finitely generated extension of $k$, $K_{r}$ is separably algebraic over $k$. It follows that $K$ itself is separably algebraic over $k$. Let $x \in K_{0}$ and choose $f$ in $k[X], X$ an indeterminate, such that $f(x)=0$ and $f^{\prime}(x) \neq 0$ where $f^{\prime}(X)=d f / d X$. If $\delta \in \Delta$,

$$
0=\delta(f(x))=f^{\prime}(x) \delta(x)+f^{\delta}(x)
$$

where $f^{\delta}$ is the result of applying $\delta$ to the coefficients of $f$. Hence $\delta(x)=$ $-f^{\delta}(x) / f^{\prime}(x)$ is in $K_{0}$. It follows that $K_{0}=K$ so that $K$ is a finitely generated extension of $k$.

Corollary. Let $f_{1}, \ldots, f_{n} \in k$ and let $U_{1}, \ldots, U_{n}$ be homogeneous linear elements of $A$ which are $\Delta$-linearly independent over $k$. Set $F_{j}=U_{j}-f_{j}$ and $q=\left[F_{1}, \ldots, F_{n}\right]$. Then $q$ is prime and $\tau(q)=m-1$ or -1 .

Before a proof of this corollary is given let us first note that although it is quite abstract it implies Janet's conjecture in its original form. In fact let us suppose $f_{1}, \ldots, f_{n}$ are functions analytic on an open connected subset $\Omega$ of $\mathbf{C}^{n}$ and that $U_{1}, \ldots, U_{n}$ are linear differential operators in $m$ variables with coefficients that are analytic on $\Omega$. Let $k$ be the field of functions meromorphic on $\Omega$ and suppose that the family of derivatives of $U_{1}, \ldots, U_{n}$ (of all orders) is linearly independent over $k$. The corollary applies and any solution $\eta_{1}, \ldots, \eta_{n}$ of the system of equations

$$
U_{j}\left(\eta_{1}, \ldots, \eta_{n}\right)=0, \quad(1 \leqslant j \leqslant n)
$$

is necessarily a zero of $q$. Two cases are possible: (1) $\tau(q)=m-1$, (2) $\tau(q)=-1$. In case (1) we can write $\chi(r)=a_{m-1} r^{m-1} /(m-1) !+\ldots$, with $a_{m-1}$ a nonzero natural number; in classical terms this is interpreted to mean that the solution of (*) depends on $a_{m-1}$ arbitrary functions of $m-1$ variables (plus possibly other functions of fewer than $m-1$ variables). In case (2) there are irreducible nonzero polynomials $P_{1}, \ldots, P_{n}$ over $k$ such that $P_{1}\left(\eta_{1}\right)=\cdots=P_{n}\left(\eta_{n}\right)=0$ for every solution $\eta_{1}, \ldots, \eta_{n}$. In particular there can only be a finite number of solutions analytic on a connected open subset of $\Omega$.

The corollary will now be proved.

It is well known (and easy to show) that $q$ is prime. Let $E=\Theta d_{A / k} F_{1}$ $\cup \cdots \cup \Theta d_{A / k} F_{n}$. Then $E$ is a linearly independent subset of the $k$-vector subspace $V$ of $\Omega_{A / k}$ spanned by $\Theta d_{A / k} y_{1} \cup \cdots \cup \Theta d_{A / k} y_{n}$. Also $\Omega_{A / k} \approx$ $A \otimes_{k} V$ so $E$ can be extended to a free basis of the $A$-module $\Omega_{A / k}$. Hence for any $\mathfrak{p}$ in $\operatorname{Spec} A, E$ is independent at $\mathfrak{p}$. This means that $F_{1}, \ldots, F_{n}$ is independent at every $\mathfrak{p}$ in $\operatorname{Spec} A$. By (2) of Theorem 2 (with $\mathfrak{p}=\mathfrak{q}$ ) we get $\tau(q)=m-1$ or -1 . 
For another application of Theorem 2 let $\mathfrak{p}=\left[y_{1}, \ldots, y_{n}\right]$. Consider elements $F_{j}=U_{j}+M_{j}$ of $A$ for $j=1, \ldots, n$ where $U_{j}$ is homogeneous linear and $M_{j} \in \mathfrak{p}^{2}$. Assume that $U_{1}, \ldots, U_{n}$ are linearly independent over $\mathscr{D}_{k}=$ $k[\Delta]$. Let $q=\left\{a \in A: a(1+\mathfrak{p}) \cap\left[F_{1}, \ldots, F_{n}\right] \neq \varnothing\right\}$ (so $q$ is the same as in the theorem). Then Theorem 2 implies $q$ is prime and $\tau(q)=m-1$ or $\left[U_{1}, \ldots, U_{n}\right]=\mathfrak{p}$. If $\tau(q) \neq m-1$, then also $q=\left[y_{1}, \ldots, y_{n}\right]$.

The talk given by Kolchin [7] in the Moscow congress of 1966 suggests it may be possible to prove a form of Janet's conjecture which is stronger than that given in Theorem 2.

\section{BIBLIOGRAPHY}

1. Maurice Janet, Sur les systemes aux dérivées partielles comprenant autant d'équations qui de fonctions inconnues, C. R. Acad. Sci. Paris 172 (1921), 1637-1639.

2. K. R. Goodearl, Global dimension of differential operator rings. II, Trans. Amer. Math. Soc. 209 (1975), 65-85.

3. Joseph Johnson, Differential dimension polynomials and a fundamental theorem on differential modules, Amer. J. Math. 91 (1969), 239-248.

4. 207-216. A notion of Krull dimension for differential rings, Comment. Math. Helv. 44 (1969),

5. Jean-Pierre Serre, Algèbre locale. Multiplicités, Lecture Notes in Math., vol. 11, SpringerVerlag, Berlin and New York, 1965.

6. Joseph Johnson, $A$ notion of regularity for differential local algebras, in Contributions to algebra, Bass, Cassidy and Kovacic, ed., Academic Press, New York, 1977, pp. 211-232.

7. Ellis Kolchin, Some problems in differential algebra, Proc. Internat. Congr. Math., (Moscow, 1966), Izdat. "Mir", Moscow, 1968, pp. 269-276.

Department of Mathematics, Rutgers University, New Brunswick, New Jersey 08903 